\title{
"L'oreille paradigmatique ». Les listes dans les manuscrits de James Joyce
}

Daniel Ferrer

\section{(2) OpenEdition}

1 Journals

Édition électronique

URL : http://journals.openedition.org/genesis/3641

DOI : 10.4000/genesis.3641

ISSN : 2268-1590

Éditeur :

Presses universitaires de Paris Sorbonne (PUPS), Société internationale de génétique artistique littéraire et scientifique (SIGALES)

Édition imprimée

Date de publication : 15 décembre 2018

Pagination : 81-92

ISBN : 979-10-231-06282

ISSN : 1167-5101

\section{Référence électronique}

Daniel Ferrer, « «L'oreille paradigmatique ». Les listes dans les manuscrits de James Joyce », Genesis

[En ligne], 47 | 2018, mis en ligne le 15 décembre 2019, consulté le 21 janvier 2021. URL : http:// journals.openedition.org/genesis/3641 ; DOI : https://doi.org/10.4000/genesis.3641 


\title{
« L'oreille paradigmatique» Les listes dans les manuscrits de James Joyce
}

\author{
Daniel Ferrer
}

$\mathrm{L}$ es listes qui figurent dans Ulysse et dans Finnegans Wake sont réputées représenter, sinon un aboutissement ${ }^{1}$, du moins une étape emblématique dans la littérature occidentale ${ }^{2}$. Le généticien est évidemment curieux d'examiner les documents qui témoignent de la confection de ces listes. Ces documents n'apportent toutefois pas de surprises majeures. On y voit à l'œuvre une dynamique d'expansion indéfinie, caractéristique bien connue de l'écriture joycienne en général ${ }^{3}$, et par ailleurs virtuellement applicable à toute liste. On trouvera en figure $1 a, b, c$ un exemple d'énumération héroï-comique poussée progressivement au-delà des limites de l'absurde, dans l'épisode du «Cyclope» d'Ulysse. On pourrait faire des constatations semblables concernant l'épisode «Ithaque» qui fait mine de réduire toute la réalité à une liste catéchistique de questions et de réponses, ellesmêmes souvent constituées de listes disproportionnées, comme celle, hyperréaliste, des objets contenus dans le tiroir de Bloom, ou celle, hyperboliquement poétique, des propriétés de l'eau qui coule au robinet, ou encore la liste (fantasmatique) des amants de Molly Bloom (fig. 2). On observe, à chaque fois que les documents de genèse sont disponibles, sur les manuscrits et les dactylogrammes, puis sur les placards et sur les multiples jeux d'épreuves, un mouvement d'extension qui semble ne jamais devoir s'arrêter. Ce débordement est au service d'une esthétique de l'excès, assumée dans le texte définitif. Contrairement à Flaubert ${ }^{4}$, Joyce n'a pas besoin d'assouvir dans l'avant-texte des pulsions expansives que l'économie narrative lui interdirait : l'économie n'est pas son propos, comme le démontrent les exemples qui viennent d'être cités.

Ces listes démesurées sont pourtant de faible ampleur si on les compare à d'autres listes qu'on rencontre dans les manuscrits. À son ami Frank Budgen, qui lui demandait un jour de 1918 comment avançait son travail sur Ulysse, Joyce répondit qu'il avait consacré toute la journée à écrire deux courtes phrases. Budgen, se souvenant de Flaubert, lui demanda s'il avait passé tout ce temps à chercher le mot juste. Pas du tout, lui répondit Joyce. Il avait tous les mots, mais c'est l'agencement qui lui posait problème, le choix parmi toutes les combinaisons possibles ${ }^{5}$. En effet, Joyce avait $l^{\prime}$ « oreille paradigmatique ${ }^{6}$ », et il savait se ménager en amont une surabondante réserve d'éléments sous forme de listes dans lesquelles il n'avait qu'à puiser les matériaux de son travail de composition. Ce sont ces listes-outils que nous allons essayer de décrire. Précisons que cette étude ne sera ni exhaustive (l'espace imparti ne permet pas d'aborder

1. Les listes ont continué de prospérer après Joyce, ne serait-ce que chez des écrivains sous forte influence joycienne, comme Beckett et Perec. On peut, dans une perspective inverse, considérer Joyce comme un auteur «perecien» par anticipation, comme le fait Jean-Michel Rabaté dans «Le calcul du doute comme pensée de l'écriture chez Joyce» (Penser, classer, écrire : de Pascal à Perec, études réunies et présentées par B. Didier et J. Neefs, Saint-Denis, Presses universitaires de Vincennes, 1990, p. 35). Voir aussi ci-dessous l'analyse des schémas de Joyce en tant que batterie de contraintes quasi oulipiennes.

2. Ainsi, Umberto Eco se propose d'étudier les listes «dans l'histoire de la littérature (de Homère à Joyce jusqu'à nos jours) ». Voir Vertige de la liste, Paris, Flammarion, 2009, p. 7.

3. Voir Daniel Ferrer et Jean-Michel Rabaté, «Paragraphes en expansion» dans L. Hay (dir.), De la lettre au livre : sémiotique des manuscrits littéraires, Paris, CNRS Éditions, 1989.

4. Selon Raymonde Debray-Genette, parlant d' «Hérodias», «il y a une grande disproportion entre la masse des notes et scénarios apographiques [...] et l'utilisation finale. L'information est abrégée et dispersée au profit du dramatisme narratif. [...] Il y a donc une sorte d'effet dissuasif de l'avant-texte documentaire qui devient l'arrière-fond mental du récit. Une des lois essentielles de cette économie documentaire peut se définir par le fait que la capacité d'impact final du document est en proportion inverse de son abondance antérieure. » («Flaubert» dans R. Debray-Genette et J. Neefs (dir.), Romans d'archives, Presses universitaires de Lille, 1987). 5. Frank Budgen, James Joyce and the Making of Ulysses, Oxford University Press, 1972, p. 20.

6. Finnegans Wake évoque «the paradigmatic ear», page 70, ligne 36. Toutes les éditions de Finnegans Wake (à l'exception de celle procurée par Danis Rose) sont identiquement paginées. Nous donnerons les références à ce texte sous la forme suivante : $F W 70.36$. 


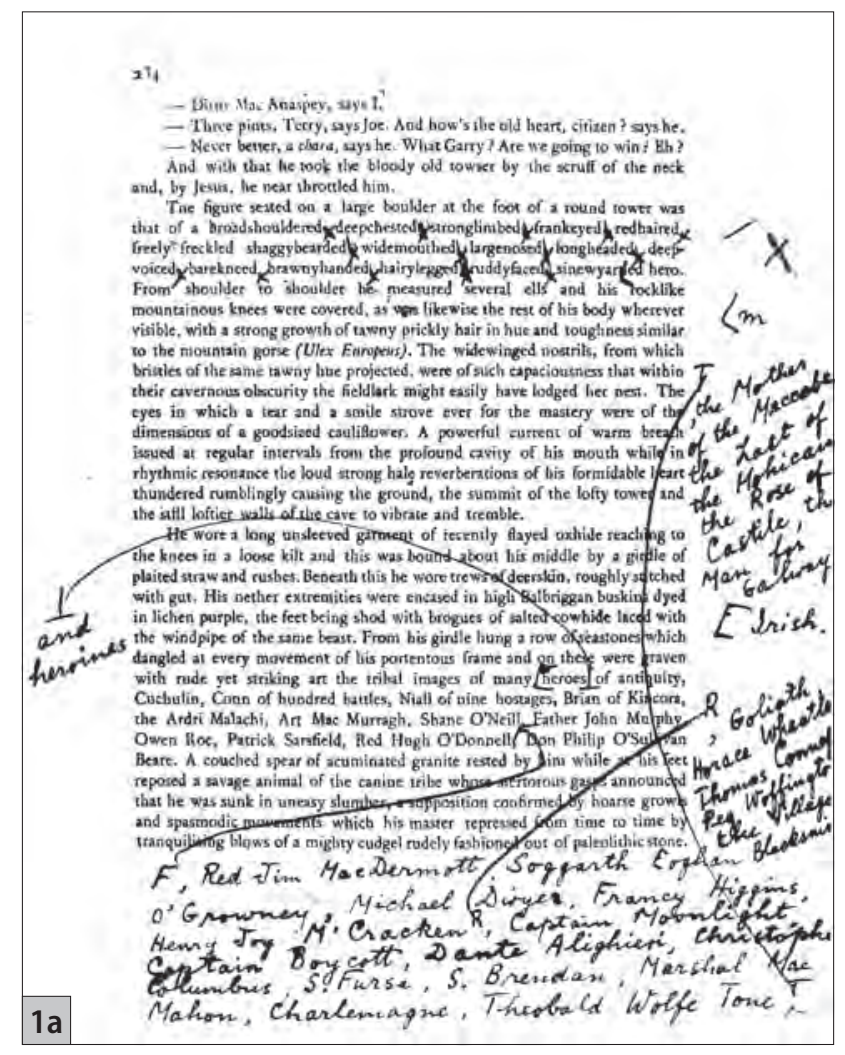

Fig. 1 : Épreuves corrigées pour l'épisode du «Cyclope» d'Ulysse. Énumération épique des «héros de l'antiquité» irlandaise dont l'effigie est accrochée à la ceinture du personnage. Au fil des ajouts sur le deuxième jeu d'épreuves, puis sur le troisième jeu, le nombre de ces «héros» passe de 13 à 88. On remarque que Joyce réclame une nouvelle épreuve, peut-être pour poursuivre l'expansion, mais la liste est demeurée telle quelle jusque dans le texte définitif. Ce qui commence en effet comme une liste de héros de l'antiquité irlandaise dérive bientôt par une série d'anachronismes, puis par des ajouts incongrus (Dante Alighieri, le dernier des Mohicans...) vers une suite burlesque, hors de toute logique.

Fig. $1 a$ et $b:$ «Cyclops». Deuxièmes épreuves (Buffalo V.C.1-18a). Avec l'aimable autorisation de la succession littéraire de James Joyce et de The Poetry Collection, Université de Buffalo. Reproduit dans le volume 25 de la James Joyce Archive, dir. Michale Groden, New York/Londres, Garland Publishing, 1978, p. 16-17. Dorénavant, les références à cette édition seront données sous la forme JJA 25 : 16-17.

Fig. 1c: «Cyclops». Troisièmes épreuves (Harry Ransom Center. The University of Texas at Austin). Avec l'aimable autorisation du Harry Ransom Center et de la succession littéraire de James Joyce. Reproduit dans JJA $25: 27$.
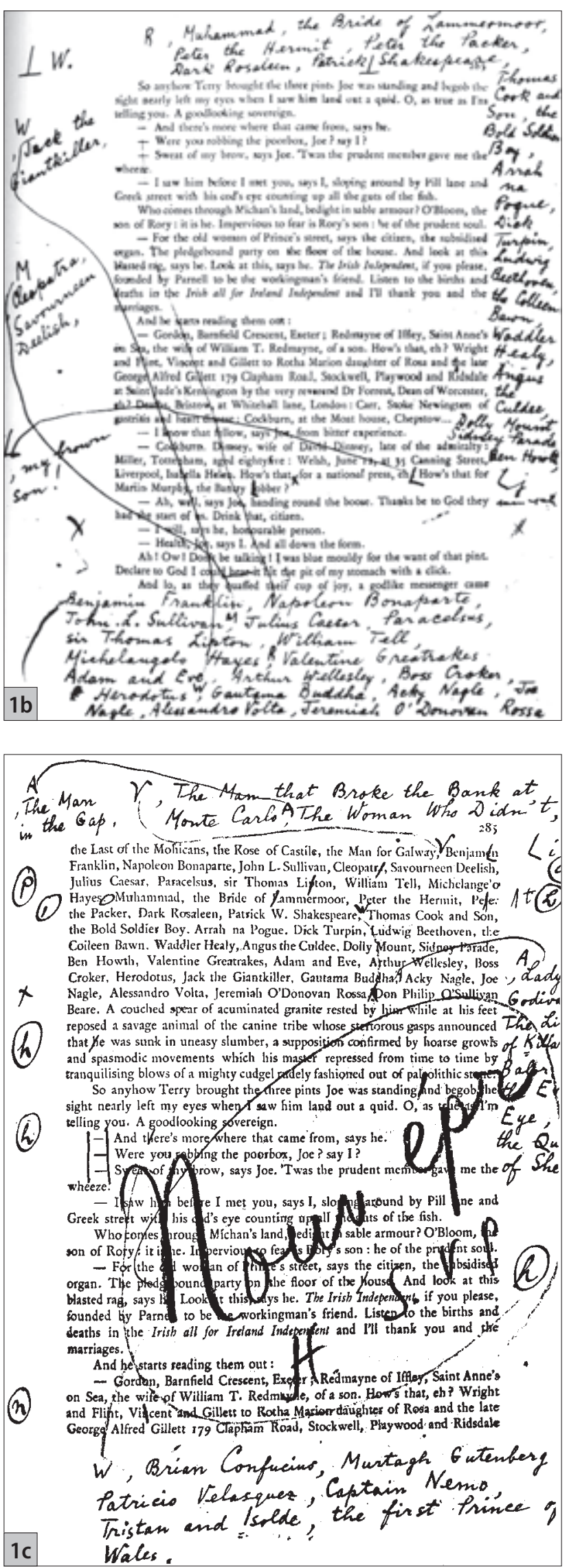


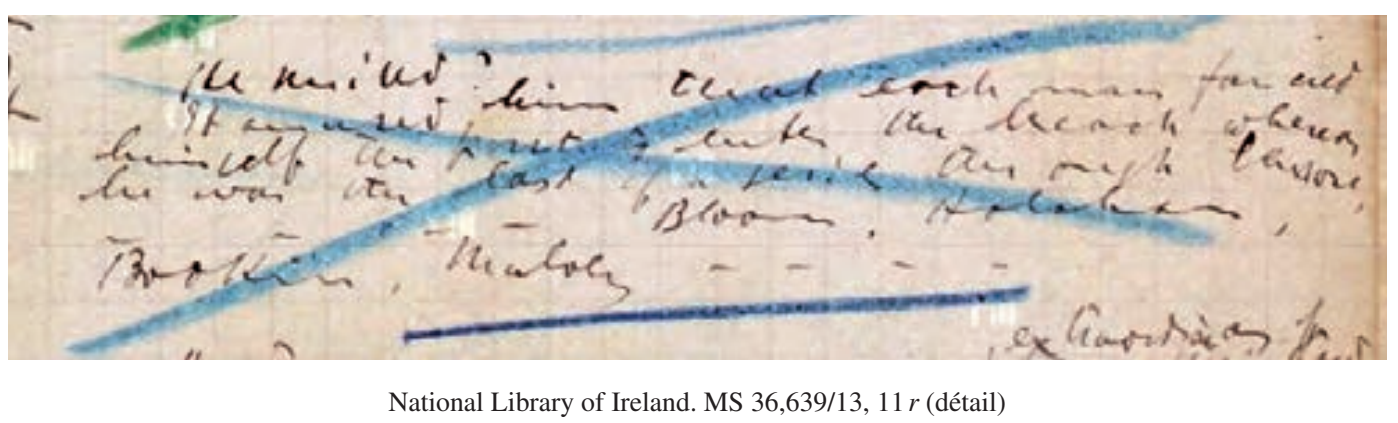

Transcription :

"He Smiled?

It amused him that each man fancied himself the first to enter the breach whereas he was the last of a series through Penrose, —— - Bloom, Holohan, Bodkin, Mulvey - - - - -

Traduction :

«Il sourit?

Cela l'amusait que chaque homme s'imagine être le premier sur la brèche alors qu'il était le dernier d'une série comprenant Penrose, ——— Bloom, Holohan, Bodkin, Mulvey - - - —

Fig. 2 : Première esquisse de la liste imaginaire des amants de Molly Bloom. Les tirets sont des tenant-lieux graphiques anticipant des noms qui restent à trouver. Ils montrent que l'important n'est pas la référence (à l'univers fictionnel) mais la production d'un effet de liste, et plus précisément de longue liste (la version définitive comportera vingt-cinq noms de supposés amants). Comme l'a fait remarquer Luca Crispi (qui le premier a attiré l'attention sur ce manuscrit dans son article «Revisiting Molly's Lovers», James Joyce Quarterly 51, 2-3, 2014, p. 489-493), certains des noms (Penrose, Bodkin) qui surgissent dans cette première version sont également des tenant-lieux provisoires, appartenant à la sphère autobiographique de Joyce (des hommes dont il avait été jaloux) et non pas à l'univers fictif d'Ulysse. Ce passage n'est lui-même qu'un item (bien délimité par des traits de couleur) de la liste des questions-réponses qui constituera l'épisode d' «Ithaque »- et qui demeure très incomplète à ce stade.

les listes antérieures à Ulysse, qui sont moins originales), ni chronologique. Nous étudierons tantôt les listes préparatoires d'Ulysse et tantôt celles de Finnegans Wake, en fonction des documents qui sont disponibles (le corpus subsistant est très vaste, mais il est loin d'être complet) et en essayant de dégager différents types.

\section{Listes structurantes et heuristiques}

Commençons par des listes qu'on pourrait qualifier de semi-publiques : elles ne figurent pas comme telles dans le texte, mais Joyce les a laissé filtrer, bien avant la publication, auprès de critiques amis pour leur servir de point d'appui et les aider à appréhender les grandes lignes d'un texte qu'ils ne savaient pas par quel bout prendre. L'impact des listes ainsi dévoilées a été extrêmement important pour la réception d'Ulysse. L'opinion d'Ezra Pound, pour lequel lecteurs et critiques ne devaient pas se préoccuper de cet «échafaudage » ayant servi à la construction et qui n'est qu'une «affaire de cuisine ${ }^{7}$ », n'a pas prévalu. Pour ce qui est de Finnegans Wake, le rôle de ces macro-listes est probablement moins important et en tout cas plus difficile à cerner, mais là aussi Joyce s'est arrangé pour en diffuser quelques-unes, telle que la liste des stades de l'histoire selon Giambattista Vico, bien avant d'avoir achevé son œuvre.

La plus connue de ces listes est celle des épisodes homériques qui correspondent aux dix-huit chapitres d'Ulysse. Le parallélisme avec l'Odyssée est annoncé dès le titre du livre et Joyce a confié très tôt l'homologie entre ses héros et ceux d'Homère. Il s'appuie sur l'Odyssée pour produire une liste de 18 titres homériques, auxquels il va faire correspondre les épisodes de son roman : Télémaque, Nestor, Protée, Calypso, Lotophages, Hadès, Éole, Lestrygons, Charybde et Scylla, Rochers flottants, Sirènes, Cyclope, Nausicaa, Bœufs du Soleil, Circé, Eumée, Ithaque, Pénélope. Nous disons qu'il produit cette liste et non qu'il la reproduit, car ces dix-huit épisodes n'apparaissent pas comme tels dans l'Odyssée, qui est organisée en Chants. Les épisodes identifiés par Joyce peuvent être à cheval sur plusieurs Chants, être enchâssés à l'intérieur d'un autre épisode, ou être simplement évoqués par Homère comme une possibilité

7. Ezra Pound, «James Joyce et Pécuchet», Mercure de France, ${ }^{\circ} 575$, 33e année, t. CLVI, 1er juin 1922, p. 307-320. 
non réalisée. L'ordre retenu pour Ulysse est différent dans une large mesure de celui de l'Odyssée. Il est attesté que Joyce avait envisagé certains autres épisodes : des notes pour un «Lacédémone» ont été retrouvées ${ }^{8}$. Les correspondances sont assez lâches, souvent astucieuses (l'aventure de Circé, qui change les hommes en pourceaux, correspond à l'épisode du bordel), parfois tirées par les cheveux. La structure d'ensemble qui en résulte est loin d'être rigide, imposant tout au plus quelques très grandes lignes : une errance qui se termine par un retour, un héros qui est précédé sur la scène du récit par son fils (ou un personnage filial)... Tout le reste est interprété librement par l'ingéniosité de Joyce. On peut même dire que cette liste, bien loin de le restreindre, lui sert à stimuler sa créativité. Il l'utilise comme une topique ${ }^{9}$, une liste de lieux communs à travers laquelle il fait passer la matière narrative dublinoise pour lui donner forme et sens. Ceci se traduit aussi bien à grande échelle - quel peut être l'équivalent des sirènes dans ce contexte? les barmaids d'un pub musical; quel peut être l'équivalent des périls de Charybde et Scylla? une discussion qui navigue entre les écueils jumeaux de l'Aristotélisme et du Platonisme... qu'au niveau de micro-correspondances ponctuelles (ainsi au début du «Cyclope» un ramoneur manque d'éborgner le narrateur de son balai), allant jusqu'à informer la substance stylistique des épisodes («Éole» est envahi par des expressions ayant trait au vent ou à l' air; le langage des «Sirènes » est soumis à une musicalisation intense en même temps que se multiplient les formules faisant allusion au son ou aux instruments de musique...).

Mais la liste homérique n'est pas seule : elle se combine avec d'autres listes, qui prétendent structurer simultanément le récit, même si elles sont intervenues plus tardivement d'un point de vue génétique, telles que la liste des organes du corps humain, la liste des arts, la liste des couleurs, la liste des techniques narratives, sans parler d'une liste de symboles maîtres... En combinant tout cela, on passe de la liste au tableau (fig. 3). Joyce en a produit plusieurs versions ${ }^{10}$, dont on ignore dans quelle mesure ils sont de véritables avanttextes ou plutôt des prospectus à destination de la critique. Dans (toutes les versions de) ce tableau, on peut constater que chaque épisode est surdéterminé par ces listes qui viennent le prendre en écharpe : par exemple, l'épisode de «Calypso», outre l'épisode homérique, correspond en même temps au rein dans la colonne des organes, à l'économie dans celle des arts, à l'orange dans celle des couleurs, à la nymphe dans celle des symboles et au «récit adulte» dans celle des techniques narratives. Si on lit le tableau horizontalement, on verra que l'épisode semble se résumer à une liste de déterminations. Joyce se serait imposé ainsi un cahier des charges, une armature de contraintes presque aussi rigide que les plus strictes contraintes oulipiennes. Dans les faits et d'un point de vue génétique, il semble que Joyce ait traité ces contraintes avec beaucoup de liberté et de légèreté. Elles se sont ajoutées progressivement comme des strates, par ajouts successifs, visant à aboutir à une saturation intense du texte qui fourmille de particules aimantées, soumises à des champs magnétiques divergents. Dans ce processus, Joyce s'est servi de matériaux qu'il avait engrangés sous la forme de listes d'un tout autre type. Ce sont ces listes, contenues principalement dans les carnets de notes (notebooks), mais aussi sur des feuillets grand format (notesheets), que nous allons étudier maintenant.

\section{Les listes d'accumulation primitive}

Parmi ces listes, il y en a qui sont, pour autant que nous le sachions, de première intention 11 , c'est-à-dire qu'elles ne sont pas constituées à partir d'autres listes antérieures mais plutôt par prélèvement à partir de textes lus par Joyce.

8. Sur ces notes, qui prennent la forme d'une curieuse liste, voir Daniel Ferrer, «An Unwritten Chapter of Ulysses? Joyce's Notes for a "Lacedemon" Episode», dans James Joyce : Whence, Whither and How. Studies in Honour of Carla Vaglio, G. Cortese, G. Ferreccio, M. T. Giaveri et T. Prudente (dir.), Alexandrie, Edizioni dell'Orso, 2015, p. 362-377 (en ligne sur le site de l'ITEM).

9. Dans le premier roman de Joyce, Portrait de l'artiste jeune homme, le sermon qui est au centre du troisième chapitre était ostensiblement construit selon un tel procédé. L'éloquent jésuite qui voulait inspirer à ses jeunes auditeurs la terreur de l'enfer passait systématiquement en revue la liste des cinq sens pour imaginer quelles pouvaient être les souffrances infligées aux damnés à travers chacun d'entre eux.

10. Voir Claude Jacquet, «Les plans de Joyce pour Ulysse», dans Ulysses cinquante ans après. Témoignages franco-anglais sur le chef-d'œuvre de James Joyce, Études anglaises, ${ }^{\circ}$ 53, Paris, Didier, 1974, p. 45-82.

11. On ne peut jamais exclure que ce que nous prenons pour des listes de première intention ait été en fait élaboré sur un support perdu, par exemple sur une feuille volante ou même au dos d'une enveloppe, mais les caractéristiques graphiques assez nettement différenciées des listes de première et de seconde intentions rendent cette hypothèse assez peu probable. 


\begin{tabular}{|c|c|c|c|c|c|c|c|}
\hline TITLE & SCENB & HOUR & ORGAN & $\mathrm{ART}$ & COLOUR & SYMBOL. & TECHNIC \\
\hline I. Telemachus & The Tower & $82 . \mathrm{m}$. & & Theology & White, gold & Heir & Narrative (young) \\
\hline 2. Nestor & The Schood & $10 \mathrm{a} . \mathrm{m}$. & & History & Brown & Horse & $\begin{array}{l}\text { Catechism (pet- } \\
\text { somal) }\end{array}$ \\
\hline 3. Proteus & The Strand & 11 a.m. & & Ehilology & Green & Tide & Monologue (male) \\
\hline 4. Calypso & The House & $8 \mathrm{am}$ & Kidney & Icomomics & Orange & Nymiph & Narrative (mature) \\
\hline 5. Lotus-eaters & The Bath & Io a.m. & Genitals & Doeany. Chemistry & & Eucharist & Narcissism \\
\hline 6. Hades & The Graveyard & II a.m. & Heart & Peligion & White, black & Caretaker & Incubism \\
\hline 7. Acolus & The Newspaper & 12 noon & Lungs & Ehetoric & Red & Editor & Enthymemic \\
\hline 8. Lestrygonians & The Lunch & I p.m. & Esophagus & Mrehitecture & & Constables & Peristaltic \\
\hline $\begin{array}{l}\text { 9. Scylla and } \\
\text { Charybdis }\end{array}$ & The Library & 2 p.m. & Brain & literature & & $\begin{array}{l}\text { Stratford, } \\
\text { London }\end{array}$ & Dialectic \\
\hline $\begin{array}{l}\text { 10. Wandering } \\
\text { Rocks }\end{array}$ & The Streets & 3 p.m. & Blood & Mechanics & & Citizens & Labyrinth \\
\hline xI. Sirens & $\begin{array}{l}\text { The Concert } \\
\text { Room }\end{array}$ & 4 p.m. & Ear & Music & & Barmaids & Fuga par earanem \\
\hline 12. Cyclops & The Tavern & 3 p.m. & Muscle & Politics & & Fenian & Gigantism \\
\hline 13. Nausicaa & The Rocks & 8 p.m. & Eye, Nose & Iainting & Grey, bluc & Virgin & $\begin{array}{l}\text { Tumescence, do } \\
\text { tumsescence }\end{array}$ \\
\hline 14. Oxen of the Sun & The Hospital & 10 p.m. & Womb & Medicine & White & Motbers & $\begin{array}{l}\text { Embryonic do- } \\
\text { velopment }\end{array}$ \\
\hline Is. Circe & The Brothel & $\begin{array}{l}12 \text { mid- } \\
\text { night }\end{array}$ & $\begin{array}{l}\text { Locomotor } \\
\text { Apparanis }\end{array}$ & Magic & & Whore & Hallucination \\
\hline 16. Eumacus & The Shelter & $t$ a.m. & Nerves & Navigarion & & Sxilors & Narrative (old) \\
\hline 17. Ithaca & The House & $2 \mathrm{anm}$ & Skeletoa & Science & & Consets & $\begin{array}{l}\text { Catechism (im- } \\
\text { persomal) }\end{array}$ \\
\hline 18. Penelope & The Bed & & Flesh & & & Earth & $\begin{array}{l}\text { Monologue } \\
\text { (female) }\end{array}$ \\
\hline
\end{tabular}

Fig. 3 : Tableau reproduit dans James Joyce’s «Ulysses», a study de Stuart Gilbert, Londres, Faber and Faber, 1930.

Parmi les carnets ayant servi à la fabrication d'Ulysse, un seul a été conservé qui contienne une liste appartenant à cette catégorie (Buffalo MS VIII.A.5) et nous disposons de la copie partielle d'un autre semblable (Buffalo MS VI.D.7), mais il paraît certain qu'il en existait de nombreux autres qui sont perdus. Pour décrire cette sorte de liste, nous nous appuierons donc principalement sur les carnets qui se rattachent à Finnegans Wake ${ }^{12}$. On peut considérer que la majorité de ceux-ci forment une liste unique et démesurée qui s'étend sur des milliers de pages. Ils présentent en effet une suite indifférenciée de brefs items (un mot ou un groupe de mots) placés les uns en dessous des autres, alternant parfois avec de courts traits de séparation, et ils s'enchaînent sans que le passage d'un carnet à l'autre ne marque aucune solution de continuité dans la prise de notes. Les éléments de cette liste proviennent de sources diverses (le plus souvent livres ou articles de journaux) mais ils sont alignés (ou plutôt empilés) pêle-mêle, sans rien qui indique leur origine (fig. 4).

Les spécialistes se sont donné pour but de distribuer cette liste en «Index »13, c'est-à-dire de déterminer l'origine de chaque terme. Pour présenter les choses autrement, ils veulent savoir quels syntagmes il a fallu détricoter pour constituer ce paradigme. Au bout de plus de quarante ans de ce jeu de piste, ils ont réussi à identifier beaucoup de sources et à produire un grand nombre de listes de notes empruntées à ces sources ${ }^{14}$, mais ces listes sont un artefact critique et, sauf exception, ne figurent pas comme telles dans

12. Les lecteurs de Genesis ont déjà pu découvrir les carnets de Finnegans Wake à travers un article paru en 1993 dans le numéro 3 (Daniel Ferrer, «Les carnets de Joyce, avant-textes limites d'une œuvre limite»), disponible en ligne. Pour plus d'information et pour une réflexion plus approfondie sur ces carnets, on se reportera à cet article, en gardant à l'esprit que la recherche a (heureusement) fait des progrès en un quart de siècle et que quelques points sont à rectifier : ainsi nous sommes maintenant sûrs que le carnet VI.A (Scribbledehobble, voir ci-dessous) est dans une large mesure un carnet de deuxième intention, postérieur à certains carnets VI.B. 13. C'est le terme souvent adopté, d'après Danis Rose, James Joyce's the Index Manuscript. Finnegans Wake Holograph Workbook VI.B.46 (Colchester, A Wake Newslitter, 1978), mais ce terme n'est sans doute pas très approprié à l'usage qu'on en fait généralement. En effet, le manuscrit étudié par Rose est bien composé d' «index», de listes identifiées par des titres, commençant toujours en haut de page, mais n'est pas du même ordre que les autres carnets répertoriés sous la cote VI.B : il s'agit à l'évidence d'un carnet de deuxième intention.

14. On en trouvera de nombreux exemples sur le site de Genetic Joyce Studies (http://www.antwerpjamesjoycecenter.com). En français, on peut consulter Claude Jacquet, Joyce et Rabelais : Aspects de la création verbale dans Finnegans Wake, Paris, Klincksieck, 1972 et Genèses de Babel : James Joyce et la création de Finnegans Wake, C. Jacquet (dir.), Paris, CNRS Éditions, 1985. 


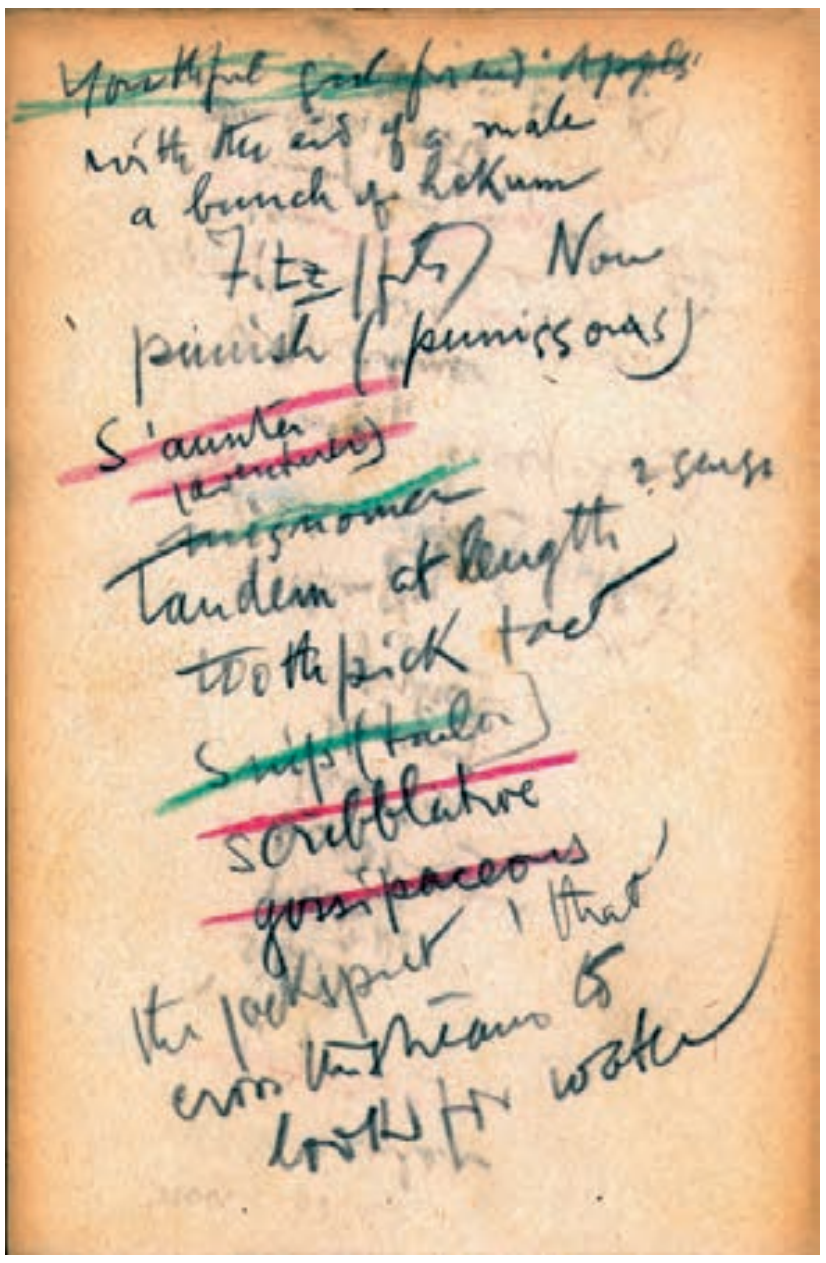

Fig. 4 : Carnet de Buffalo VI.B.6, p. 90. La plupart des notes de cette page proviennent de l'ouvrage d'Otto Jespersen The Growth and Structure of the English Language (1923). Avec l'aimable autorisation de la succession littéraire de James Joyce et de The Poetry Collection, University at Buffalo.

les carnets où les entrées sont mélangées, coupées de leur source et totalement décontextualisées.

Les éléments de ce vaste ensemble ne se différencient ostensiblement ni par leur origine, ni par l'utilisation pour laquelle ils sont prévus. Il est clair que ce monstrueux paradigme réunit des éléments que Joyce pensait susceptibles d'être intégrés à son prochain texte. C'est là sa raison d'être et sa seule unité. Mais comme il n'avait d'abord aucune idée de ce que serait ce prochain ouvrage ${ }^{15}$, puis pendant longtemps une idée assez vague de sa consistance, cette intentionnalité demeure la plupart du temps très floue. Ceci est confirmé, nous le verrons, par le recyclage des notes inutilisées qui est opéré dans les listes de deuxième ou de énième intention.

On peut certes isoler quelques sous-listes à l'intérieur de ce vaste ensemble. Ainsi, dans les carnets VI.B.29 et VI.B.24, qui se suivent chronologiquement, on trouve des notes (une suite de toponymes urbains), provenant pour l'essentiel des articles de l'Encyclopaedia Britannica consacrés aux grandes villes du monde et de quelques autres sources d'histoire urbaine, qui sont destinées à nourrir la dernière partie du chapitre 3 du livre IV de Finnegans Wake, où HCE, le héros, se présente comme fondateur et bâtisseur de ville(s). Individualisée à la fois par son origine et par sa destination, concentrée sur un même support, on peut considérer qu'il s'agit bien d'une sous-liste caractérisée, même si elle ne se présente pas de manière parfaitement consécutive (elle est souvent interrompue par des notes disparates).

Plus complexe est le cas du recensement des rivières et fleuves, qui ne revêt pas la forme canonique de la liste, puisqu'il est dispersé tout au long du corpus des carnets. Les noms de cours d'eau, puisés au fil des années et au hasard des lectures, sont identifiés par la lettre « $\mathrm{R} »$, ce qui permet de reconstituer le paradigme dispersé. Ces rivières ont donc une multitude de sources, mais elles sont toutes destinées à se déverser dans un unique réservoir, le célèbre chapitre 8 , «Anna Livia Plurabelle».

Voici comment Umberto Eco présente les choses :

Autre énumération vertigineuse de lieux, celle de Joyce dans le chapitre de Finnegans Wake connu sous le titre «Anna Livia Plurabelle» où, pour rendre le sens du courant de la rivière Liffey, il a inséré, diversement masqués sous des puns et des mots-valises, des centaines de noms de fleuves de tous les pays. Le lecteur peine à reconnaître des fleuves pour la plupart inconnus, tels que Chebb, Futt, Bann, Duck, Sabrainn, Till, Waag, Bomu, Boyana, Chu, Batha, Skollis, Shari, Sui, Tom, Chef, Syr Darya ou Ladder Burn, etc. [...] Ce qui rend cette liste potentiellement infinie, ce n'est pas seulement l'effort que doit faire le lecteur pour identifier tous les fleuves, c'est aussi un double soupçon : les critiques ont identifié plus de fleuves que Joyce n'en a introduits, ou bien, la combinatoire du langage en crée plus que ce que pensaient les critiques et Joyce. On a du mal à classer ce type de liste. Il s'agit d'une liste par gloutonnerie, par topos de l'ineffabilité (on ne peut dire combien de fleuves et de rivières existent au monde), par

15. Sur les tâtonnements des débuts de Finnegans Wake, voir L. Crispi et S. Slote (dir.), How Joyce Wrote Finnegans Wake. A Chapter-by-Chapter Genetic Guide, Madison, University of Wisconsin Press, 2007 et, en français, Daniel Ferrer «Par où (re)commencer, ou de Tristan à Finnegan », dans James Joyce, Brouillons d'un baiser. Premiers pas vers Finnegans Wake, édition et présentation de Daniel Ferrer, préface et traduction de Marie Darrieussecq, Paris, Gallimard, coll. «Du monde entier», 2014. 
pur amour de la liste. Joyce a travaillé des années pour recenser tous les noms de fleuves et de rivières, avec la collaboration de plusieurs personnes, sans poursuivre de but géographique 16 .

Nous sommes obligés de corriger Eco sur un point, important pour notre sujet : «Anna Livia Plurabelle», contrairement à plusieurs autres chapitres de Finnegans Wake, ne contient pas de liste et ne se présente pas comme une énumération. Pour l'écrire, Joyce a certes, comme nous venons de le voir, utilisé une collection de noms de rivières accumulée dans ses carnets. Le critique, ou même le simple lecteur un peu obsessionnel, peut certes essayer de reconstituer une liste des (noms de) cours d'eau qu'il trouve dans le chapitre (sachant, comme le suggère Eco, que les listes des différents lecteurs ne seront pas identiques et ne coïncideront certainement pas avec celle de Joyce), mais le chapitre luimême, dialogue animé entre deux lavandières, ne s'égrène pas comme le chapelet d'une liste, il coule, tout en fluidité, à l'inverse de l'effet staccato des nombreuses énumérations qui figurent dans Ulysse et Finnegans Wake et dont il a été question au début de cet article. Il semble qu'Eco opère une projection quelque peu abusive de l'avant-texte et de ce que nous savons de la genèse du texte sur le texte lui-même.

On peut citer d'autres cas de sous-listes constituées dans un but précis, par exemple des notes à partir d'ouvrages sur les jeux enfantins pour le chapitre II.1, consacré aux jeux des enfants de HCE, mais dans l'ensemble la liste court, indifférenciée, d'un bout à l'autre des carnets. Pour orienter ses notes vers l'univers de son œuvre en cours d'élaboration, Joyce a inventé une série de sigles dont il a affecté certains items, mais cette orientation reste peu contraignante et ne concerne qu'une faible partie de la liste ${ }^{17}$.

\section{Listes de répartition et de recyclage}

Le processus de transfert direct depuis une liste de première intention vers les brouillons n'est pas toujours observé dans la genèse de Finnegans Wake et n'est pas le cas le plus fréquent dans la genèse d'Ulysse. Après avoir accumulé les matériaux pêle-mêle, sous forme de listes indifférenciées, Joyce les regroupe souvent sous forme de listes spécifiques pourvues d'un intitulé. Il en est (au moins) de trois sortes : $a$ ) listes thématiques, $b$ ) listes épisodiques et, $c$ ) les plus étranges, que j'appellerais listes rétrospectives-prospectives.
Dans les carnets ou cahiers, les items de ces listes secondaires sont le plus souvent placés à la suite les uns des autres et séparés par deux points (équivalents, dans la ponctuation anglaise, à notre point-virgule), tandis que dans les Notesheets ils sont placés en colonnes, les uns en dessous des autres.

a) $\mathrm{Si}$ on exclut les carnets de jeunesse, du type livre de raison, les principales listes thématiques sont contenues dans un carnet qui a été intitulé par les chercheurs «Subject notebook», car les matériaux y sont listés par sujets. Chaque page est consacrée à un personnage ou à un thème d'Ulysse : Simon, Stephen, Leopold, la théosophie, la rhétorique, les Irlandais et les Juifs (sur deux pages en vis-à-vis)... Apparemment unique en son genre, il est antérieur aux carnets épisodiques dont nous allons parler maintenant.

b) Joyce semble avoir abandonné assez vite ce type de regroupement de son matériau par sujets pour adopter un regroupement par épisodes. Après avoir utilisé la liste des épisodes homériques comme plan général du livre, il s'en sert comme d'une grille pour ranger ses matériaux. Chaque carnet est divisé en sections d'une ou plusieurs pages portant chacune le titre d'un épisode. Ces sections se succèdent dans un ordre aléatoire, sans rapport avec l'ordre de succession des épisodes dans le roman. Il s'agit d'une démarche qui semble naturelle : les notations sont regroupées en fonction de leur destination, c'est-à-dire des chapitres où elles doivent être intégrées. Mais si la liste des épisodes homériques a permis à Joyce de fixer assez tôt le déroulement général du livre, en tant que grille de répartition, elle est loin d'indiquer une distribution figée.

La circulation qui s'établit entre les diverses listes est intense. Il n'est pas rare qu'un élément passe de carnet en carnet, de carnet en notesheet, de notesheet en notesheet, de notesheet en carnet. Si nous prenons une des notesheets, marquée d'un grand $« \mathrm{C} »$ pour indiquer qu'elle est consacrée à l'épisode «Circé» (fig. 5), nous constatons que les éléments de la liste qu'elle contient proviennent d'au moins trois carnets différents et de quatre autres notesheets. Ils seront utilisés dans deux carnets, une notesheet et deux

16. Vertige de la liste, op. cit., p. 81-82

17. Pour une description et une analyse du rôle de ces sigles, on se reportera à l'article paru dans Genesis, $\mathrm{n}^{\circ} 3$ ( «Les carnets de Joyce, avant-textes limites d'une œuvre limite», op. cit., p. 52-53). 
brouillons différents ${ }^{18}$. Dans certains cas ce dispatching semble suivre une simple logique de transfert : par exemple l'item «Thursday's child has far to go (SD)», emprunté à une comptine enfantine, apparaît d'abord dans le « Subject notebook» où il est listé sous la rubrique «Stephen». Il sera bien utilisé dans le premier brouillon de «Circé» à propos de S[tephen] D[edalus] et il ne changera pas jusqu'au texte définitif (U 15.3687). En revanche, l'entrée «H. M. as fireman or D.M.P. » provient d'une notesheet consacrée à l'épisode $\mathrm{du}$ «Cyclope» et aboutira dans un carnet sous la rubrique «Lotophages », avant d'être effectivement incorporée dans l'épisode des «Lotophages ». Les items migrent d'une liste à l'autre, d'un épisode à l'autre (et même d'une œuvre à l'autre, puisque des listes qui avaient été compilées pour Portrait de l'artiste en jeune homme ont été réutilisées pour Ulysse, des listes prévues pour Ulysse ont été recyclées dans le chantier de Finnegans Wake).

c) L'existence de ce qu'on peut appeler les listes rétrospectives-prospectives renforce l'idée que les regroupements par épisodes ne sont pas simplement une affaire de logistique. Ces listes se rencontrent dans un (très gros) carnet préparatoire de Finnegans Wake connu sous le nom de Scribbledehobble ${ }^{19}$. Pendant un certain temps, Joyce y a regroupé des items, provenant pour la plupart des carnets de première intention que nous avons décrits précédemment, selon une grille préétablie modelée sur ses œuvres antérieures : son recueil de poèmes Musique de chambre, les différentes nouvelles de Gens de Dublin, les cinq parties du Portrait, les trois actes des Exilés et les dix-huit épisodes d'Ulysse figurent en tant qu'intitulés, en tête de page et sur la table des matières ${ }^{20}$. Il est clair qu'il ne s'agit plus d'assigner une destination aux items listés sous ces rubriques. Les épisodes homériques («Télémaque», «Nestor», «Protée»...) ne figurent plus dans cette liste en tant que tels mais bien en tant qu'épisodes de la création joycienne. Le classement n'est pas orienté vers le futur du texte, mais vers le passé de l'œuvre. Tout se passe comme si le matériau devait subir un stage de pré-assimilation, traverser un sas d'acclimatation à travers l'œuvre déjà écrite avant d'être admise dans l'œuvre future. On peut penser qu'aux yeux de Joyce, chacun de ses écrits antérieurs est porteur d'une couleur et d'un projet artistique qui lui est propre. L'œuvre à venir doit revêtir toutes ces couleurs et subsumer tous ces projets. Chaque item de la liste des œuvres devient donc à son tour une catégorie sous laquelle va se ranger une liste d'éléments présentant des affinités avec lui (selon des critères qui ne sont pas toujours clairs pour nous). Par exemple, sous le titre de la nouvelle «Les Sœurs» vont être disposés des items ayant trait aux contes et à la narration. Il semble qu'il s'agisse de préparer une palette de tonalités familières dans laquelle l'artiste pourra puiser. Il est clair en tout cas que le listage et le re-listage des éléments destinés à être utilisés dans l'œuvre ne se résument pas à un transfert, neutre et sans reste.

\section{L'usage des listes}

Lorsque les éléments de la liste sont prélevés, soit pour être insérés directement dans les brouillons, soit pour être intégrés dans d'autres types de listes de deuxième ou de énième intention, ils sont rayés avec des crayons de couleurs différentes pour éviter qu'ils ne soient utilisés deux fois, chaque couleur indiquant une campagne de prélèvement distincte 21 . Cette façon de rayer ce qui a été utilisé fait songer à une liste de courses sur laquelle on coche ou on biffe les items au fur et à mesure qu' on les range dans le panier à commissions, mais ce serait une analogie trompeuse. Le statut pragmatique de la liste, ou des listes, constituées par les carnets de Joyce ne s'apparente pas à celui de la liste de courses, qui stipule des achats à effectuer (le but de la liste étant précisément de n'en oublier aucun), et moins encore à celui d'une recette de cuisine (protocole opératoire, définissant une suite d'actions à exécuter dans l'ordre précis de leur énumération), mais bien plutôt, pour rester dans le domaine alimentaire, à celui d'une carte de restaurant : une liste de plats proposés pour composer un menu, un éventail de choix qui ne sont pas totalement neutres (les prix indiqués

18. Je remercie Luca Crispi de m'avoir communiqué un travail encore inédit sur cette notesheet. Je m'appuie également sur ses nombreux et précieux travaux d'analyse et de classification de l'avant-texte d'Ulysse. 19. C'est sous ce titre que Thomas E. Connolly a publié le carnet de Buffalo VI.A. : Scribbledehobble : the Ur-Workbookfor Finnegans Wake, Evanston, Northwestern University Press, 1961.

20. Cette table des matières est reproduite dans l'article de Genesis, ${ }^{\circ} 3$, avec d'autres pages des carnets de Joyce.

21. Sans systématisme, toutefois. Il semble que Joyce attrapait simplement à chaque fois le crayon de couleur qu'il avait sous la main, sans que ces couleurs aient une signification précise. 


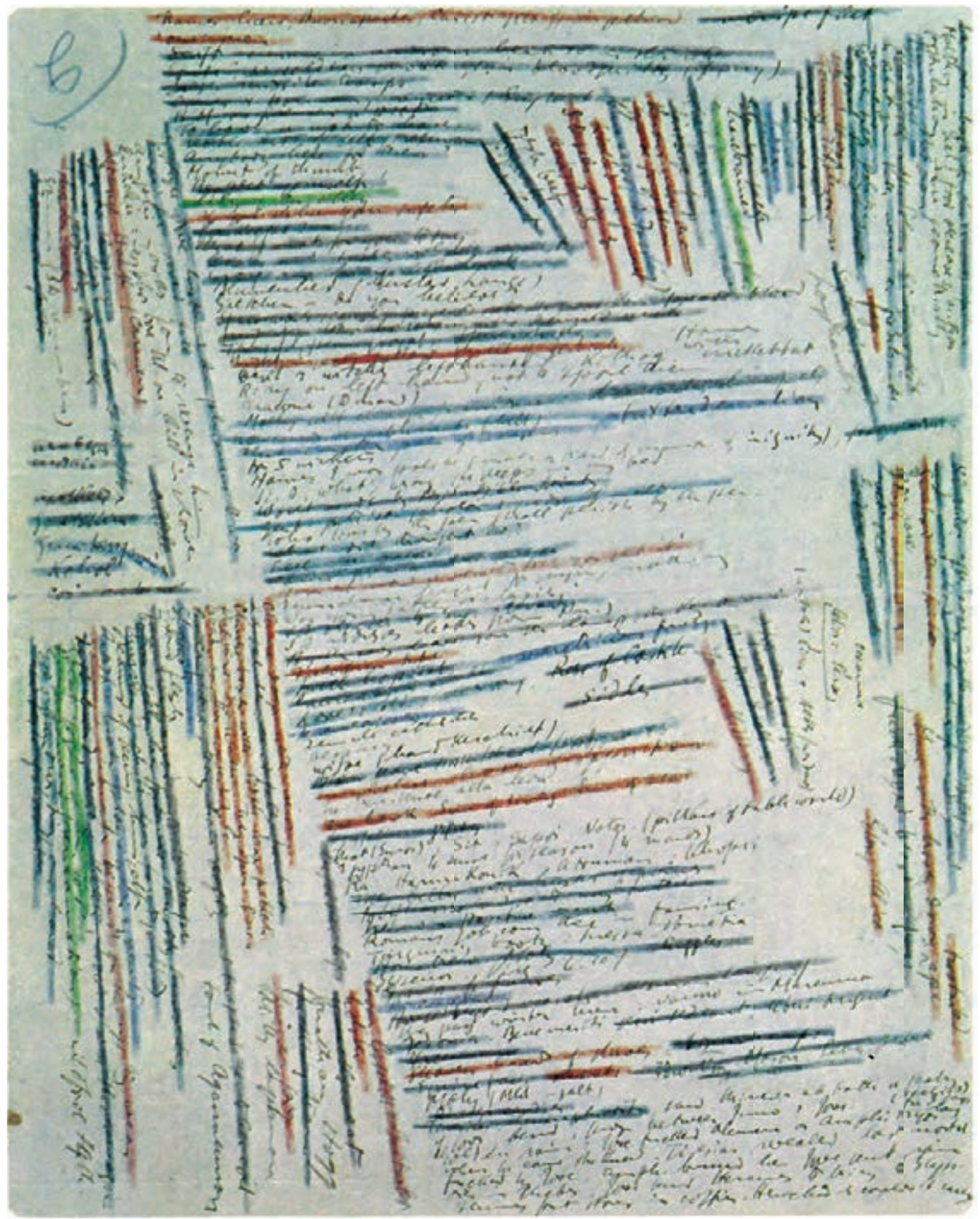

Fig. 5 : «Circe» notesheet British Library Add ms 49975. (c) British Library Board.

Avec l'aimable autorisation de la British Library et de la succession littéraire de James Joyce. Reproduit dans JJA 12:45.

L'entrée «Thursday's child has far to go (SD)» est inscrite verticalement au bas de la marge de gauche.

L'entrée «H. M. as fireman or D.M.P.» est inscrite dans la colonne centrale. Les deux items sont rayés au crayon bleu. 
orientent la sélection, des astérisques désignent les plats qui peuvent entrer dans la formule économique...), ni dépourvus de contraintes (on ne peut pas, théoriquement, prendre un dessert en entrée), mais où aucun item n'est imposé.

Le mode opératoire de Joyce était le suivant : à chaque étape de l'écriture de ses brouillons et à chaque strate de ses révisions, il parcourait ses carnets et y butinait les entrées susceptibles d'être introduites dans le travail en cours, recouvrant la texture de base de broderies et de sur-broderies. Le paradigme ne défile pas en un point du syntagme, comme dans le fonctionnement habituel de la langue, quand on cherche le mot juste, propre à occuper la position qu' on lui assigne dans la phrase. Le paradigme est ici promené le long du syntagme, jusqu'à ce qu'un accord se fasse, jusqu'à ce qu'une rencontre se produise, jusqu'à ce qu'une disponibilité du signifiant, une valence libre, permette à un des items du paradigme de venir s'accrocher au syntagme, ou jusqu'à ce qu'une ouverture sémantique rende possible une expansion qui permettra de loger un item. Dans Ulysse, c'est souvent la souplesse du monologue intérieur et de ses associations libres qui ouvre d'infinies possibilités d'expansion; dans Finnegans Wake, c'est la plasticité d'un langage affranchi des limitations du dictionnaire et du monolinguisme qui offre à chaque pas la chance de coïncidences heureuses, disponibles pour accueillir les ajouts provenant des carnets.

Ainsi dans «Anna Livia Plurabelle», le dialogue des deux lavandières est ponctué de marques d'impatience, l'une des commères pressant l'autre de poursuivre son récit malveillant. Dès le premier jet (JJA 48:7 ; BL 47471b-76), un paragraphe se termine par l'injonction «Go on, go on!» («Continue, continue!»). Après plusieurs strates de brouillons, une mise au net et de nombreux dactylogrammes, Joyce, profitant d'une ressemblance phonique et rythmique, y substitue un élément de son paradigme fluvial : "Garonne, garonne !» (JJA 48:125; BL 47474-175). Notons que cette substitution doit s'analyser en fait comme une addition, car les mots «Go on! go on! » continuent de se faire entendre sous le bruissement du fleuve français. Bien plus tard (JJA 48:354; 47475-83), au bout de plusieurs pré-publications du passage et de nombreux jeux d'épreuves, Joyce insérera en amont les mots : «I amstel waiting» (I am still waiting, «j'attends toujours », où l'on entend aussi la rivière Amstel). C'est sous cette forme que l'injonction apparaîtra dans le texte définitif de Finnegans Wake, lestée d'un fleuve hollandais et d'un fleuve français : «I amstel waiting. Garonne, garonne!» (FW 205.15).

Dans un cas comme celui-ci, il apparaît clairement que c'est le paradigme qui a engendré le fragment de syntagme (comme dans un poème, une rime peut avoir engendré le vers qui la contient). C'est une parfaite illustration de la définition jakobsonienne de la fonction poétique : le principe d'équivalence, qui est constitutif de l'axe de la sélection, se projette sur l'axe de la combinaison (la similarité se projette sur la contiguïté, ce qui reste habituellement virtuel se projette dans l'actuel) - la liste se projette sur le texte et le suscite. Cependant, la formule de Jakobson ne suffit pas à décrire le fonctionnement du texte joycien, car l'axe de la sélection ne se contente pas de se projeter, il s'effondre sur lui-même. Si choisir, c'est éliminer tous les autres choix possibles, on constate que la sélection ne s'opère pas : «amstel» est à la fois verbe, adverbe et fleuve. Dans un mot comme «sanglorians» ( $F W$ 04.07), il y a à la fois sanglot riant, sang gloire, sans gloire, sangle or, Saint Lawrence, Angles et Aryens... et toutes ces lectures font sens dans le cadre du début de Finnegans Wake. Il apparaît que, du fait de ce refus du choix, chaque point de la chaîne verbale est occupé par toute une liste de morphèmes superposés qui viennent former des liaisons multiples avec les items des listes voisines.

Dans le grand syntagme que constitue le déroulement d'Ulysse comme dans chaque phrase de Finnegans Wake, chaque position est occupée par une sorte de paradigme écrasé, par une liste dont tous les éléments s'actualisent simultanément. D'un point de vue génétique cela ne veut évidemment pas dire que toutes les couches de ce feuilletage soient apparues simultanément. Elles sont le résultat d'un travail minutieux de complexification progressive, mais aussi d'une attention aux ambiguités du signifiant, d'une attitude ouverte et accueillante envers les hasards de la signification. Ceci est rendu possible grâce à la mise en œuvre de toute une batterie de listes-outils combinées. Je me suis efforcé de décrire les différents types de liste et l'usage que Joyce en fait, d'Ulysse à Finnegans Wake, mais cet usage ne reste pas totalement identique au fil du temps, en fonction de l'évolution du projet esthétique, et aussi sans doute de la 
plus grande maîtrise acquise par Joyce avec l'expérience, qui lui permet de se contenter d'instruments moins élaborés.

Si nous revenons sur Ulysse et la suite des épisodes homériques (qui n'est rappelons-le, que l'une des listes qui se combinent dans le tableau-maître) telle que Joyce l'élabore, on s'aperçoit que cette liste sert à la fois à l'inventio (en faisant office de topique très productive), à la memoria (en offrant une grille permettant de ranger les matériaux, de les mettre en attente et de les retrouver le moment voulu), à la dispositio (elle donne la structure générale) et enfin à l'elocutio (en venant gauchir la texture même des épisodes qui, au fil des étapes de la genèse, se trouve de plus en plus surchargée d'allusions homériques).

Pour Finnegans Wake, où c'est clairement l'elocutio qui prime, où la texture acquiert une complexité extrême, au détriment relatif de la structuration, Joyce adopte progressivement un dispositif simplifié (après l'étrange tentative du Scribbledehobble), se contentant pour l'essentiel d'une gigantesque liste primaire dans laquelle il puise au fur et à mesure de ses besoins, par campagnes successives. C'est ainsi qu'il obtient le maximum de souplesse nécessaire à la mise en œuvre de son projet ultime. 
DANIEL FERRER est chercheur à l'ITEM (ENS-CNRS). Il a écrit des livres et des articles sur Joyce, Woolf, Faulkner, Poe, Stendhal, Flaubert, Zola, Barthes, Cixous, sur la peinture, les humanités numériques, la génétique cinématographique et la théorie de la critique génétique. Ouvrages récents : Logiques du brouillon. Modèles pour une critique génétique (Le Seuil, coll. «Poétique», 2011); Renascent Joyce (Gainesville, University Press of Florida, 2013) et l'édition de Brouillons d'un baiser : premiers pas vers Finnegans Wake (Gallimard, coll. «Du monde entier», 2014).

daniel.ferrer@ens.fr

\section{Les listes dans les manuscrits de James Joyce}

Les célèbres listes d'Ulysse et de Finnegans Wake sont issues de remarquables manuscrits, témoignant d'un mouvement irrépressible d'expansion. Mais les manuscrits de Joyce recèlent des listes plus remarquables encore, par leurs dimensions comme par leur diversité, qui n'apparaissent pas dans les œuvres publiées. On peut distinguer des listes structurantes et heuristiques (y compris la fameuse liste des épisodes homériques), des listes d'accumulation primaires et des listes de répartition et de recyclage. Joyce puise dans l'immense réservoir constitué par ces listes pour enrichir son texte, voire pour le susciter, projetant, selon la formule jakobsonienne, le paradigme sur l'axe syntagmatique, mais poussant si loin le travail de surdétermination que chaque point de la chaîne syntagmatique tend à devenir un paradigme condensé, une liste effondrée sur elle-même.

The celebrated lists in Ulysses and Finnegans Wake have been elaborated on remarkable manuscripts revealing an irrepressible movement of expansion. Among Joyce's manuscripts, however, we can find lists that do not appear in the published works and that are even more remarkable, because of their size and because of their diversity. There are structuring and heuristic lists (including the famous list of Homeric episodes), lists of primitive accumulation and lists for distribution and recycling. These lists form a huge bank from which Joyce draws at will to enrich or even to generate his texts, projecting, according to Roman Jakobson's definition of the poetic function, the paradigm onto the syntagmatic axis. The work of overdetermination is carried to such an extent that each point of the syntagmatic chain tends to become a concentrated paradigm, a self-contained list.

Die berühmten Listen von Ulysses und Finnegans Wake stammen aus bemerkenswerten Manuskripten und zeugen von einer unbändigen Expansionsbewegung. Aber Joyce's Manuskripte enthalten noch bemerkenswertere Listen, sowohl hinsichtlich ihrer Größe als auch ihrer Vielfalt, die in den veröffentlichten Werken nicht erscheinen. Man kann unterscheiden zwischen strukturierenden und heuristischen Listen (einschließlich der berühmten Liste der homerischen Episoden), primäre Akkumulationslisten und Verteilungs- bzw. Recyclingslisten. Joyce schöpft aus dem enormen Reservoir, das diese Listen bilden, um seinen Text zu bereichern, ja, um ihn zu erschaffen, wobei er nach der Jakobson 'schen Formel das Paradigma auf die syntagmatische Achse projiziert, aber die Arbeit der Überdeterminierung so weit treibt, dass jeder Punkt der syntagmatischen Kette dazu neigt, ein verdichtetes Paradigma zu werden, eine Liste, die in sich selbst zusammenbricht.
Las célebres listas del Ulises y de Finnegan Wake forman parte de manuscritos extraordinarios, que dan cuenta de un movimiento incontenible de expansión. Pero los manuscritos de Joyce contienen listas todavía más extraordinarias, tanto por sus dimensiones como por su diversidad, que no aparecen en las obras publicadas. Se pueden distinguir las listas estructurantes y heurísticas (incluida la famosa lista de episodios homéricos), las listas de acumulación primarias y las listas de repartición y de reciclado. Joyce recurre a ese inmenso depósito constituido por las listas para enriquecer su texto, e incluso para suscitarlo, proyectando, según la fórmula jakobsoniana, el paradigma en el eje sintagmático, pero llevando tan lejos el trabajo de sobredeterminación, que cada eslabón de la cadena sintagmática tiende a convertirse en un paradigma condensado, una lista derrumbada sobre sí misma.

As célebres listas de Ulysses e Finnegans Wake provêm de notáveis manuscritos, que reflectem um irreprimível movimento de expansão. Mas os manuscritos de Joyce guardam listas ainda mais notáveis, tanto pelas dimensões como pela diversidade, que não figuram nas obras publicadas. Entre elas, listas estruturantes e heurísticas (tais como a famosa lista dos episódios homéricos), listas básicas de acumulação e listas de distribuição e reciclagem. Joyce serve-se do imenso reservatório constituído por essas listas para enriquecer o seu texto, ou mesmo para o conceber, projectando, segundo a fórmula jakobsoniana, os paradigmas sobre o eixo dos sintagmas, elevando esse trabalho de determinação a tal extremo que cada elo da cadeia sintagmática se transforma em paradigma condensado, em lista empilhada sobre si mesma.

Le celebri liste di Ulisse e Finnegans Wake traggono origine da manoscritti notevoli che testimoniano un incontenibile movimento di espansione. I manoscritti di Joyce, tuttavia, custodiscono liste ancor più notevoli, sia per dimensione sia per diversità, che non appaiono nelle opere pubblicate. Tra queste possiamo distinguere le liste strutturanti ed euristiche (compresa la famosa lista degli episodi omerici), le liste d'accumulazione primarie e le liste di ripartizione e di riciclaggio. Joyce attinge all'immensa riserva costituita da queste liste per arricchire il suo testo e anche per suscitarlo, proiettando, secondo la formula di Jakobson, il paradigma sull'asse sintagmatico, ma spingendo il lavoro di sovradeterminazione così lontano che ogni punto della catena sintagmatica tende a diventare un paradigma condensato, una lista che contiene se stessa. 